\title{
Readability and suitability assessment of educational materials in promoting the quality of life for postmenopausal women
}

\author{
Mohammad Ali Morowatisharifabad ${ }^{1}$, Nooshin Yoshany², Manoj Sharma ${ }^{3}$, Narjes Bahri ${ }^{4}$, \\ Sara Jambarsang ${ }^{5}$
}

${ }^{1}$ Department of Health Education and Promotion, School of Public Health, Shahid Sadoughi University of Medical Sciences, Yazd, Iran 2Department of Health Education and Health Promotion, Social Determinants of Health Research Center, School of Public Health, Shahid Sadoughi University of Medical Sciences, Yazd, Iran

${ }^{3}$ Environmental \& Occupational Health, School of Public Health, University of Nevada, Las Vegas, United States

${ }^{4}$ Department of Midwifery, Faculty of Medicine, Social Development and Health Promotion Research Center, Gonabad University

of Medical Sciences, Gonabad, Iran

${ }^{5}$ Department of Bio-Statistics and Epidemiology, School of Public Health, Shahid Sadoughi University of Medical Sciences, Yazd, Iran

\begin{abstract}
Introduction: Educational materials are frequently used by health providers to inform postmenopausal women about menopause acceptance behavior. However, little attention has been paid to the readability and suitability of these educational materials. The study aimed to determine the readability and suitability of educational materials in promoting the quality of life for postmenopausal women.

Material and methods: Multiple instructional materials and books were used for the design and preparation of educational materials and were then tailored to the target group. Readability was measured by using the readability assessment of materials (RAM); and suitability was determined by the suitability assessment of materials (SAM) that considers characteristics such as content, graphics, layout/topography, and cultural appropriateness. Twenty reviewers, including 10 postmenopausal women, 5 postmenopausal women family members, and 5 health experts scored the educational materials.

Results: The mean readability score \pm standard deviation (SD) of the educational materials was $10 \pm 1.6$ and $8 \pm 1.4$, for the pamphlet and daily activities' booklets, respectively, which were increased to $14 \pm 0.6$ and, $16 \pm 1.3$, after tailoring the content. The average SAM scores before and after tailoring the content were $45 \%$ for the pamphlet, which was increased to $81 \% ; 45 \%$ for the daily activities' booklets, which was increased to $86 \%$. The increase in all scores was significant $(p<0.001)$. The final tailored educational material was rated "superior media" on the SAM ratings.

Conclusions: Given that most of the printed materials are suitable for people with higher education levels, health providers are strongly advised to prepare simple and understandable education materials that may increase the likelihood of consumer perception and recall.
\end{abstract}

Key words: menopause, postmenopause, quality of life, comprehension, suitability, pamphlet, booklet, health literacy, readability.

\section{Introduction}

Menopause, as a natural phenomenon and a physiological transition in middle-aged women, is individually, socially, and culturally important. It is associated with complications and consequences that can affect women's quality of life and weaken the good and healthy feeling in them [1]. Health education interventions are one of the alternative strategies for promoting health and coping with menopausal symptoms [2]. Studies have shown that the severity of the menopaus- al symptoms is associated with low educational levels, low socioeconomic status, race, frequency of deliveries, and high age at the menopause onset [3]. Therefore, the acceptance and management of menopausal symptoms and the enhancement of women's health literacy have been considered. Health literacy is the ability to obtain, read, understand, and use the information in order to make appropriate health decisions and follow instructions for treatment [4]. Increasing people's knowledge and health literacy can help them in facing 
health problems [5]. Poor health is an independent risk factor for low use of preventive services, higher hospital admission, medication non-adherence, and mortality, and is a consequence of inadequate health literacy [6]. Studies have shown that more than one-third of adults have inadequate proficiency in health literacy tasks [7]. Primary sources of health information are physicians and health professionals, but for reasons such as lack of time, large numbers of clients, high medical costs and poor communication skills, these specialists are not always able to meet patients' information needs [8]. Health information resources in different formats such as pamphlets, booklets, brochures, and with a variety of purposes such as developing self-care skills, increasing awareness about diseases, and increasing awareness of the availability of medical and health facilities are used as a complementary component in training programs $[9,10]$. Educational materials are frequently used by health providers and have advantages such as portability and consistency [11]. Much of this information is forgotten, when only oral information is provided. Thus health providers are encouraged to prepare printed educational material for people to reinforce and supplement the information that has been provided orally [12]. Audience comprehension is the main goal of educational resource producers. In the process of understanding, people first receive different input stimuli and information and send it to the brain; secondly, these recorded stimuli are processed. Thirdly, this information is understood by making some neurochemical changes in the brain. if the received stimuli in the first step not properly recorded, the second and third stages do not occur and therefore the understanding is not achieved $[13,14]$. Some standards are being established to enhance the quality of patient education materials, such as recommendations to write educational materials at a sixth-grade reading level [15]. The instruments used to assess the appropriateness of printed material are readability and suitability. Readability refers to the ease or difficulty of reading an educational medium. However, readability alone is not enough for improving perception. Suitability offers a systematic method to objectively assess the suitability of health information materials for a particular audience in a short time [16].

Most postmenopausal women do not have adequate information about menopausal symptoms and are not aware of the strategies to control their side effects [17-19]. The first step in promoting the women's compatibility and compliance with the menopausal changes is to enhance their awareness about the physiological process and the body functions. Awareness about the signs and symptoms of menopause can help women to live a comfortable and happy life. It also alleviates their psychological problems [20]. In 1990, the world's population of women over 50 was 467 million, which is estimated to increase to 1,200 million by 2030
[21]. In 2011, there were 7,539,293 women aged 40-60 years in Iran [22]. Symptoms observed in postmenopausal women include hot flashes, sweating, palpitations, sleep disorders, irritability, lethargy, depressed mood, forgetfulness, decreased libido, depression, vaginal dryness, painful intercourse, urinary symptoms, memory reduction, decreased concentration, restlessness, as well as joint and bone problems [23]. The increasing population of elderly in Iran and prevalence of all kinds of health problems and menopausal symptoms in these ages can affect work, social activity, enjoyment of life, leisure time, sleep and quality of life of women and their families and endanger the health integrity and health of postmenopausal women [24]. So it is necessary to use educational materials to educate post-menopausal women about the healthy life styles that can affect the severity of menopause symptoms. For all target groups, printed educational materials for health issues will be useful, if it is understood by the recipient [25]. Little attention has been paid to the readability and suitability of these educational materials for postmenopausal women. Assessment of written educational materials about menopausal hormone therapies showed that most of the materials are written at a high reading level [26]. In some studies conducted in Yazd regarding menopausal health education, the used media for intervention has not been evaluated [27-29].

While results from the National Adult Literacy showed that one in four adults in the United States have low reading level [30], the readability and suitability of education materials for postmenopausal women remains relatively unexplored. To address this gap, the specific aim of this study was to determine the readability and suitability of education materials in promoting the quality of life for postmenopausal women.

\section{Material and methods}

\section{Study design and population}

This study was conducted in 2019 (14 September - 22 October) in Yazd, located in the center of Iran. The participants enrolled in this study were 15 postmenopausal aged between 45-55 years old, 5 postmenopausal women family members ( 3 husbands and 2 children), and 5 health experts ( 2 health education specialists and 3 educational technologists). In order to select 15 postmenopausal women, three health care centers were selected randomly in Yazd city. Then participants were chosen randomly from the list of the clients. In this study, printed educational materials, included pamphlets about improving menopause quality of life and daily activities' booklets to monitor daily activities. In order to evaluate the appropriateness of the prepared printed educational material, readability and suitability tools were used. The readability of the ma- 
terial was assessed by the "readability assessment of materials" (RAM) and suitability was accessed through the "suitability assessment of materials" (SAM). Initially, the educational materials were evaluated technically by the experts. Then, according to their points of view, the necessary changes were made and the materials were tailored according to the target group. Tailored material was then returned to the target group and the suitability and readability were assessed again.

\section{Readability assessment of materials}

RAM assesses the difficulty of reading an educational medium in three parts, which are having a specialized content (range of scores 0-6), misspelling (range of scores 0-6), and typographical mistakes (range of scores $0-6$ ). The range of scores in media readability assessment is from 0 to 18 and the acceptable score is more than 10 [31].

\section{Suitability assessment of materials}

Suitability was measured using the SAM created by Doak et al. [15]. The SAM is a systematic tool to assess printed health-related educational resources in a short amount of time. The SAM has been validated [32] and successfully used in prior studies of other printed health information $[33,34]$. The SAM consists of 6 evaluation criteria: content (e.g., "behavior information to help solve their problem"), literacy demand (e.g., "common, explicit words are used"), graphics (e.g., "simple, adult-appropriate, line drawings/sketches are used"), layout and typography (e.g., "type size is at least 12 point, no ALL CAPS for long headers or running text"), learning stimulation and motivation (e.g., "complex topics are subdivided into small parts so that readers may experience small successes in understanding or problem solving"), and cultural appropriateness (e.g., "images and examples present the culture in positive ways").

Three readers were trained in the SAM scoring techniques. Each reviewer scored all study materials and was blinded to the source of the material. The mean SAM scores were used in the analysis. The SAM consists of 22 items grouped under six factors, namely content, literacy demands, graphics, layout and typography, learning stimulation and motivation, and cultural appropriateness. Each of the 22 items is rated in terms of the degree to which it meets set criteria, on an ordinal scale of $0,1,2$, and not applicable, where $0=$ inadequate, 1 = adequate, and 2 = superior. Scores are summed to yield an overall raw score for the material. This is converted to a percentage of the possible total score for that material, with $70-100 \%$ being considered to be superior material, 40-69\% adequate material, and 0-39\% not suitable material [15].

\section{Statistical analysis}

The Kolmogorov-Smirnov (KS) test was used to check the normality of quantitative variables and showed that the data had a normal distribution. The collected data were analyzed by SPSS software version 22. Descriptive statistics were reported for all variables. The mean score of SAM and RAM before and after tailoring was compared using paired t-tests. The level of significance in the tests was considered less than 0.05 .

\section{Ethical approval of studies and trial registration}

Ethical approval for this study has been obtained by the ethics committee affiliated with Shahid Sadoughi University of Medical Sciences, Yazd, Iran (reference number IR.SSU.SPH.REC.1397.137) and date (02/02/2019). Registration of this randomized control trial has been completed with the Iranian Registry of Clinical Trials, IRCT20190206042640N1.

\section{Results}

Out of 20 participants in the study, 15 people were postmenopausal aged between 45-55 years old, 5 people were postmenopausal women family members ( 3 husbands and 2 children), and 5 people were health experts ( 2 health education specialists and 3 educational technologists).The mean age of the women were $51 \pm 2.43$. Among the participants $85 \%$ were women, $60 \%$ of women were in high school or less education level (Table 3).

Table 1 . Readability assessment of materials (RAM)

\begin{tabular}{|c|c|c|c|c|}
\hline Criteria/scores & 0 & 2 & 4 & 6 \\
\hline $\begin{array}{l}\text { Being professional } \\
\text { and practical } \\
\text { (difficulty of content) }\end{array}$ & $\begin{array}{l}\text { Fully specialized } \\
\text { content }\end{array}$ & $\begin{array}{l}\text { Specialized content in } \\
\text { simple language }\end{array}$ & $\begin{array}{l}\text { Specialized content in } \\
\text { simple language and } \\
\text { somewhat practical }\end{array}$ & $\begin{array}{l}\text { Specialized content in simple } \\
\text { language focusing on their } \\
\text { applicability }\end{array}$ \\
\hline Writing status & $\begin{array}{l}\text { Over } 4 \text { writing } \\
\text { problems }\end{array}$ & $\begin{array}{l}3 \text { to } 4 \text { writing } \\
\text { problems }\end{array}$ & $\begin{array}{l}1 \text { to } 2 \text { writing } \\
\text { problems }\end{array}$ & No problem writing \\
\hline Having typed mistakes & $\begin{array}{c}\text { More than } 4 \text { typed } \\
\text { mistakes }\end{array}$ & 3 to 4 typed mistakes & 1 to 2 typed mistakes & No typed mistakes \\
\hline
\end{tabular}


Table 2. Suitability assessment of materials (SAM)

\begin{tabular}{ll}
\hline Factors to be rated & Score \\
\hline 1. Content \\
(a) Purpose is evident \\
2 Purpose is explicitly stated in title, or cover illustration, or introduction \\
1 Purpose is not explicitly. It is implied, or multiple purposes are stated \\
0 No purpose is stated in the title cover illustration, or introduction \\
\hline
\end{tabular}

(b) Content about behaviors

2 Thrust of the material is application of knowledge/skills aimed at

1 Desirable reader behavior rather than non-behavior facts

0 Nearly all topics are focused on non-behavior facts

(c) Scope is limited

2 Scope is limited to essential information directly related to the purpose. Experience shows

it can be learned in time allowed.

1 Scope is expanded beyond the purpose; no more than 40 percent is non-essential information.

Key reports can be learned in time allowed

0 Scope is far out of proportion to the purpose and time allowed

(d) Summary or review included

2 A summary is included and retells the key messages in different words

and examples

1 Some key ideas are reviewed.

0 No summary or review is included

\section{Literacy demand}

(a) Reading grade level

$25^{\text {th }}$-grade level or lower (5 years of schooling level)

$16^{\text {th }}$, $7^{\text {th }}$, or $8^{\text {th }}$-grade level (6-8 years of schooling level)

$09^{\text {th }}$-grade level and above (9 years or more of schooling level)

(b) Writing style, active voice

2 Both factors:

(1) Mostly conversational style and active voice

(2) Simple sentences are used extensively; few sentences contain embedded information

1 Both factors:

(1) About 50 percent of the text uses conversational style and active voice

(2) Less than half the sentences have embedded information

0 Both factors:

(1) Passive voice throughout

(2) Over half the sentences have extensive embedded information

(c) Vocabulary uses common words

2 All three factors:

(1) Common words are used nearly all of the time

(2) Technical, concept, category, value judgment (CCVJ) words are explained by examples

(3) Imagery words are used as appropriate for content

1 (1) Common words are frequently used

(2) Technical and CCVJ words are sometimes explained by examples

0 Two or more factors:

(1) Uncommon words are frequently used in lieu of common words

(2) No examples are given for technical and CCVJ words

(3) Extensive jargon

(d) Context is given first

2 Consistently provides context before presenting new information

1 Provides context before new information about 50 percent of the time

0 Context is provided last or no context is provided

(e) Learning aids via "road signs," subtitles and captions

2 Nearly all topics are preceded by an advance organizer (a statement that tells what is coming

next)

1 About 50 percent of the topics are preceded by advance organizers

0 Few or no advance organizers are used

\section{Graphics}

(a) Cover graphic shows purpose

2 The cover graphic is:

(1) friendly

(2) attracts attention

(3) clearly portrays the purpose of the material to the intended audience

1 The cover graphic has one or two of the superior criteria

0 The cover graphic has none of the superior criteria 
Table 2. Cont.

(b) Type of graphics

2 Both factors:

(1) Simple, adult-appropriate, line drawings/sketches are used

(2) Illustrations are likely to be familiar to the viewers

1 One of the superior factors is missing

0 None of the superior factors are present

(c) Relevance of illustrations

2 Illustrations present key messages visually so the reader/viewer can grasp the key ideas from

illustrations alone. No distractions

1 (1) Illustrations include some distractions

(2) Insufficient use of illustrations

0 One factor:

(1) Confusing or technical illustrations (non-behavior related)

(2) No illustrations, or an overload of illustrations

(d) Lists and tables explained

2 Step-by-step directions, with an example, are provided that will build comprehension and self-efficacy

1 "How-to" directions are too brief for reader to understand and use the graphic without additional counseling

0 Graphics are presented without explanation

(e) Captions used for graphics

2 Explanatory captions with all or nearly all illustrations and graphics

1 Brief captions used for some illustrations and graphics

0 No captions

4. Layout and typography

(a) Layout factors

2 At least 5 of the following 8 factors are present:

- Illustrations are on the same page adjacent to the related text

- Layout and sequence of information are consistent, making it easy for the patient to predict

the flow of information

- Visual cuing devices (shading, boxes, and arrows) are used to direct attention to specific points

or key content

- Adequate white space is used to reduce appearance of clutter

- Use of color supports and is not distracting to the message. Viewers need not learn color codes

to understand and use the message

- Line length is 30-50 characters and spaces

- There is high contrast between type and paper

- Paper has non-gloss or low-gloss surface

1 At least three of the superior factors are present

0 (1) Two (or less) of the superior factors are present

(2) Looks uninviting or discouragingly hard to read

(b) Typography

2 The following 4 factors are present:

- Text type is in uppercase and lowercase serif (best) or sans-serif

- Type size is at least 12 point

- Typographic cues (bold, size, color) emphasize key points

- No ALL CAPS for long headers or text

1 Two of the superior factors are present

0 One or none of the superior factors are present or six or more type styles and sizes are used on a page

(c) Subheads ("chunking') used

2 (1) Lists are grouped under descriptive subheadings or "chunks"

(2) No more than five items are presented without a subheading

1 No more than seven items are presented without a subheading

0 More than seven items are presented without a subheading

5. Learning stimulation, motivation

(a) Interaction used

2 Problems or questions presented for reader responses

1 Question-and-answer format used to discuss problems and solutions (passive interaction)

0 No interactive learning stimulation provided

(b) Behaviors are modeled and specific

2 Instruction models specific behaviors or skills (for example, for nutrition instruction, emphasis

is given to changes in eating patterns or shopping or food preparation/cooking tips; tips to read labels)

1 Information is a mix of technical and common language that the reader may not easily interpret

(e.g., technical: starches -80 calories per serving; high fiber -1 to 4 grams of fiber in a serving)

0 Information is presented in nonspecific or category terms such as the food groups 
Table 2. Cont.

\begin{tabular}{lc}
\hline Factors to be rated & Score \\
\hline (c) Motivation, self-efficacy & Comments \\
2 Complex topics are subdivided into small parts so that readers may experience small successes \\
In understanding or problem-solving, leading to self-efficacy \\
1 Some topics are subdivided to improve the readers' self-efficacy \\
0 No partitioning is provided to create opportunities for small successes \\
\hline
\end{tabular}

\footnotetext{
6. Cultural appropriateness

(a) Match in logic, language, experience (LLE)

2 Central concepts/ideas of the material appear to be culturally similar to the LLE of the target

culture

1 Significant match in LLE for 50 percent of the central concepts

0 Clearly a cultural mismatch in LLE
}

(b) Cultural image and examples

2 Images and examples present the culture in positive ways

1 Neutral presentation of cultural images or foods

0 Negative image such as exaggerated or caricatured cultural characteristics, actions, or examples

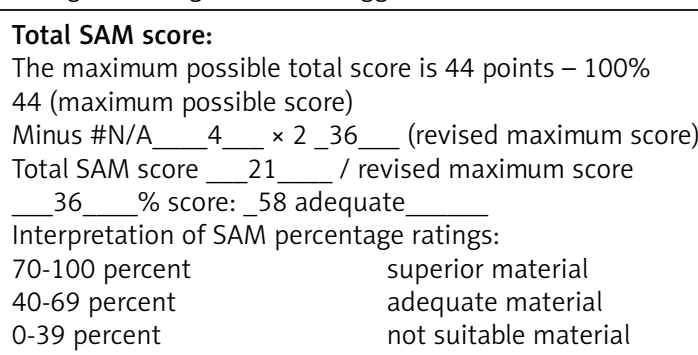

Scoring: 2 points for superior rating, 1 point for adequate rating, 0 points for not suitable rating, N/A if the factor does not apply to this material

Out of 20 participants in the study, 15 people were postmenopausal aged between 45-55 years old, 5 people were postmenopausal women family members ( 3 husbands and 2 children), and 5 people were health experts ( 2 health education specialists and 3 educational technologists).The mean age of the women were $51 \pm 2.43$. Among the participants $85 \%$ were women, $60 \%$ of women were in high school or less education level (Table 3).

The mean readability score \pm standard deviation (SD) of the educational materials were $10 \pm 1.6$ and $8 \pm 1.4$, for the pamphlet and daily activities' booklets, respectively, which were increased to $14 \pm 0.6$ and, $16 \pm 1.3$, after tailoring the content (Table 4).

The average SAM scores before and after tailoring the content were $45 \%$ for the pamphlet, which was increased to $81 \%$. The increase in all scores was significant $(p<0.001)$. Those findings showed the final educational material was "superior media" on the SAM ratings (Table 5).

The average SAM score for daily activities' booklets was $45 \%$, which significantly was increased to $86 \%$ after tailoring the content. Those findings showed the final educational material was "superior media" on the SAM ratings (Table 6).

\section{Discussion}

Annually large budgets are being spent in the production of educational materials but little attention is paid to the appropriateness of these materials for the target group. The pamphlet in this study addressed a va-
Table 3. Demographic characteristics of participants

\begin{tabular}{lll}
\hline Variable & \multicolumn{2}{c}{ Data } \\
\cline { 2 - 3 } & Mean & SD \\
\hline Age (years) & 51 & 2.43 \\
\hline Women & 57 & 3.01 \\
\hline Family member (husbands) & 24 & 2.35 \\
\hline Family member (children) & 46 & 5.76 \\
\hline Health experts & $n$ & $(\%)$ \\
\hline Educational level & & \\
\hline Women & 9 & 60 \\
\hline High school or less & 3 & 20 \\
\hline Diploma & 3 & 20 \\
\hline Associate degree & & \\
\hline Family member (husbands) & 2 & 66.67 \\
\hline High school or less & 1 & 33.33 \\
\hline Diploma & & \\
\hline Family member (children) & 100 \\
\hline Diploma & 50 \\
\hline Bachelor & & \\
\hline Health experts & & \\
\hline PhD & & \\
\hline
\end{tabular}

Table 4. Mean score of the readability assessment of materials before and after tailoring

\begin{tabular}{lcc}
\hline Readability score & \multicolumn{2}{c}{ Materials } \\
\cline { 2 - 3 } & Pamphlet & Daily activities' booklet \\
\hline Before & $10 \pm 1.6$ & $8 \pm 1.4$ \\
\hline After & $14 \pm 0.6$ & $16 \pm 1.3$ \\
\hline
\end{tabular}


Table 5. Results of the assessment pamphlet suitability assessment of materials (SAM) score check list before and after tailoring

\begin{tabular}{|c|c|c|c|}
\hline SAM item and description & Score before tailoring & Score after tailoring & $p$-value \\
\hline 1. Content & & & $<0.001$ \\
\hline a. Purpose is evident & $0.80 \pm 0.61$ & $1.60 \pm 0.50$ & \\
\hline b. Content about behavior & $0.70 \pm 0.65$ & $1.75 \pm 0.44$ & \\
\hline c. Scope is limited & $0.80 \pm 0.61$ & $1.70 \pm 0.47$ & \\
\hline d. Summary or review included & $0.70 \pm 0.47$ & $1.60 \pm 0.50$ & \\
\hline \multicolumn{4}{|l|}{ 2. Literacy demand } \\
\hline a. Reading grade level & $0.65 \pm 0.48$ & $1.65 \pm 0.48$ & \\
\hline b. Writing style, active voice & $0.80 \pm 0.61$ & $1.65 \pm 0.48$ & \\
\hline c. Vocabulary uses common words & $1.10 \pm 0.64$ & $1.70 \pm 0.47$ & \\
\hline d. Context is given first & $0.90 \pm 0.55$ & $1.75 \pm 0.44$ & \\
\hline e. Learning aids via "road signs" & $0.90 \pm 0.55$ & $1.50 \pm 0.51$ & \\
\hline \multicolumn{4}{|l|}{ 3. Graphics } \\
\hline a. Cover graphic shows purpose & $1.00 \pm 0.56$ & $1.65 \pm 0.48$ & \\
\hline b. Type of graphics & $1.10 \pm 0.71$ & $1.60 \pm 0.50$ & \\
\hline c. Relevance of illustrations & $1.10 \pm 0.64$ & $1.55 \pm 0.51$ & \\
\hline d. List, tables, etc. explained & $1.00 \pm 0.72$ & $1.45 \pm 0.51$ & \\
\hline e. Captions used for graphics & $1.30 \pm 0.57$ & $1.60 \pm 0.50$ & \\
\hline \multicolumn{4}{|l|}{ 4. Layout and typography } \\
\hline a. Layout factors & $0.90 \pm 0.55$ & $1.55 \pm 0.51$ & \\
\hline b. Typography & $0.75 \pm 0.44$ & $1.60 \pm 0.50$ & \\
\hline c. Subheads (chunking) used & $0.85 \pm 0.48$ & $1.85 \pm 0.36$ & \\
\hline \multicolumn{4}{|l|}{ 5. Learning stimulation, motivation } \\
\hline a. Interaction used & $1.05 \pm 0.60$ & $1.60 \pm 0.50$ & \\
\hline b. Behaviors are modeled and specific & $1.15 \pm 0.48$ & $1.85 \pm 0.36$ & \\
\hline c. Motivation-self-efficacy & $1.15 \pm 0.58$ & $1.75 \pm 0.44$ & \\
\hline \multicolumn{4}{|l|}{ 6. Cultural appropriateness } \\
\hline a. Match in logic, language, experience & $0.95 \pm 0.51$ & $1.80 \pm 0.41$ & \\
\hline b. Cultural image and examples & $0.75 \pm 0.44$ & $1.65 \pm 0.48$ & \\
\hline Total score earned by SAM & 20 & 36 & \\
\hline Percentage points earned by SAM & 45 & 81 & \\
\hline
\end{tabular}

riety of topics including the definition of menopause, menopause symptoms, Common complications during menopause, Useful strategies to reduce menopausal symptoms and daily activities' booklet is a notebook that a postmenopausal woman should tick her daily activities. A study by Abdolmalaki et al. in Iran found that a high percentage of postmenopausal women (48.7\%) do not have good health literacy. It should be noted that women need to have enough information to judge whether their symptoms need to be treated and how to monitor medical care [35]. So consideration of the level of health literacy of the target group is essential in the preparation of educational materials.

The present study aimed to determine the readability and suitability of postmenopausal women education material in promoting the quality of life. Readability refers to the ease of comprehension of printed material with respect to its writing style [36] and suitability assessment can be used to evaluate printed materials against factors known to enhance people's understanding of printed materials [15]. In this study, analysis of suitability indicated that pamphlet and daily activities' booklets were in the adequate range before the tailoring that enhanced to superior range after tailoring, while analysis of readability showed pamphlet was in acceptable range before and after tailoring but daily activities' booklets wasn't in acceptable range before tailoring that enhanced after tailoring. This finding is consistent with results from the Sadeghi et al. study in Iran about the readability and suitability assessment of adolescent education materials in preventing hookah smoking [37], Hoffmann et al. in Australia about assessing the suitability of written stroke materials: an evaluation of the interrater reliability of the SAM checklist [12] and Rhee et al. in the USA about the educational material about rheumatic diseases [16]. However, the results of Walsh 
Table 6. Results of the assessment daily activities' booklets suitability assessment of materials (SAM) score check list before and after tailoring

\begin{tabular}{|c|c|c|c|}
\hline SAM item and description & Score before tailoring & Score after tailoring & $p$-value \\
\hline 1. Content & & & $<0.001$ \\
\hline a. Purpose is evident & $0.90 \pm 0.47$ & $1.70 \pm 0.41$ & \\
\hline b. Content about behavior & $0.65 \pm 0.58$ & $1.75 \pm 0.44$ & \\
\hline c. Scope is limited & $0.65 \pm 0.58$ & $1.80 \pm 0.41$ & \\
\hline d. Summary or review included & $0.65 \pm 0.67$ & $1.70 \pm 0.47$ & \\
\hline \multicolumn{4}{|l|}{ 2. Literacy demand } \\
\hline a. Reading grade level & $0.75 \pm 0.55$ & $1.75 \pm 0.44$ & \\
\hline b. Writing style, active voice & $1.00 \pm 0.72$ & $1.70 \pm 0.47$ & \\
\hline c. Vocabulary uses common words & $1.10 \pm 0.64$ & $1.70 \pm 0.47$ & \\
\hline d. Context is given first & $0.65 \pm 0.58$ & $1.75 \pm 0.44$ & \\
\hline e. Learning aids via "road signs" & $0.80 \pm 0.61$ & $1.65 \pm 0.48$ & \\
\hline \multicolumn{4}{|l|}{ 3. Graphics } \\
\hline a. Cover graphic shows purpose & $1.05 \pm 0.60$ & $1.75 \pm 0.44$ & \\
\hline b. Type of graphics & $1.10 \pm 0.55$ & $1.65 \pm 0.48$ & \\
\hline c. Relevance of illustrations & $1.25 \pm 0.63$ & $1.65 \pm 0.48$ & \\
\hline d. List, tables, etc. explained & $0.65 \pm 0.67$ & $1.60 \pm 0.50$ & \\
\hline e. Captions used for graphics & $1.15 \pm 0.58$ & $1.70 \pm 0.47$ & \\
\hline \multicolumn{4}{|l|}{ 4. Layout and typography } \\
\hline a. Layout factors & $0.80 \pm 0.41$ & $1.65 \pm 0.48$ & \\
\hline b. Typography & $0.90 \pm 0.55$ & $1.60 \pm 0.50$ & \\
\hline c. Subheads (chunking) used & $0.90 \pm 0.55$ & $1.80 \pm 0.41$ & \\
\hline \multicolumn{4}{|l|}{ 5. Learning stimulation, motivation } \\
\hline a. Interaction used & $1.10 \pm 0.64$ & $1.80 \pm 0.41$ & \\
\hline b. Behaviors are modeled and specific & $1.20 \pm 0.52$ & $1.80 \pm 0.41$ & \\
\hline c. Motivation-self-efficacy & $1.10 \pm 0.55$ & $1.60 \pm 0.50$ & \\
\hline \multicolumn{4}{|l|}{ 6. Cultural appropriateness } \\
\hline a. Match in logic, language, experience & $1.05 \pm 0.51$ & $1.85 \pm 0.36$ & \\
\hline b. Cultural image and examples & $0.75 \pm 0.44$ & $1.65 \pm 0.48$ & \\
\hline Total score earned by SAM & 20 & 38 & \\
\hline Percentage points earned by SAM & 45 & 86 & \\
\hline
\end{tabular}

and Volsko in the USA about the readability assessment of internet-based consumer health information [38], is not consistent with this study and the educational material assessed in their study did not have proper readability. SAM instrument offers a systematic method to objectively assess the suitability of health information materials for a particular audience in a short time [38]. In this study, the SAM mean score was increased after correction and tailoring the content. Hoffmann et al.'s study in Australia about assessing the suitability of written stroke material was in line with our findings and showed printed materials enhance people's understanding [12]. Finnie et al. in a systematic review showed only two of the seven Cancer Education Print and Web-based materials were suitable [39]. In this study, the average SAM score for pamphlet and daily activity booklet was $45 \%$, before the tailoring, which is considered "ade- quate" on the SAM ratings also in Weintraub et al. study titled an evaluation of the written education materials for patients with prostate cancer, the mean overall SAM rating was "adequate." However, average scores were higher (63.3\%) in comparison to our study [34]. The mean readability level of materials in Eames et al. study titled "The Suitability of Written Education Materials for Stroke Survivors and Their Carers was (grade 9) higher than participants" mean reading ability (grade 7-8) [40], while the mean score of readability of pamphlet in this study was acceptable ( $>10)$ that is not consistent with Eames's study but This finding is consistent with results from the Sadeghi et al. study [37]. Thus modifying printed educational material and tailoring them according to the target group is important for facilitating learning and recall of the information. A limitation of this study was the small sample of participants $(n=20)$ 
which may not be representative of all postmenopausal women and their needs.

Proper planning should be done for the production and distribution of educational media in accordance with the readability and suitability standards. Also, health educators who prepare education materials should be trained for this goal.

\section{Conclusions}

We recommend that guidelines for designing educational materials should be established. Health educators should strive to ensure that the quality of the printed education materials is appropriate for the target group and also should evaluate the printed educational materials by doing pilot study prior to dissemination. SAM checklist can be used to identify the proportion of education materials provided to the target group and also RAM checklist can determine the readability level of the target group. The findings showed that the printed educational materials were enhanced after evaluation by the RAM and the SAM checklist and they were in accordance with the characteristics of the postmenopausal women. Compliance with these recommendations may increase the likelihood of consumer perception and recall.

\section{Acknowledgments}

The authors would like to thank the research deputy of Shahid Sadoughi University of Medical Sciences, which financially supported this research and all of the individuals who participated in this study because of their valuable help.

\section{Disclosure}

The authors report no conflict of interest.

\section{References}

1. Sheikhan Z, Ozgoli G, Azar M, et al. Prevalence of sexual violence among infertile women. Adv Nursing Midwifery 2014; 23: 33-40.

2. Rotem M, Kushnir T, Levine R, Ehrenfeld M. A Psycho Educational Program for improving women's attitudes and coping with menopause symptoms. J Obstet Gynecol Neonatal Nurs 2005; 34: 233-240.

3. Friedman SH, Sajatovic M, Schuermeyer IN, et al. Menopause-related quality of life in chronically mentally ill women. Int J Psychiat Med 2005; 35: 259-271.

4. Berkman ND, Davis TC, McCormack L. Health literacy: what is it? J Health Commun 2010; 15 (S2): 9-19.

5. Sadeghi R, Mazloomy S, Hashemi M, Rezaeian M. The effects of an educational intervention based on the health belief model to enhance HIVpreventive behaviors among male barbers in Sirjan. J Rafsanjan Univ Med Sci 2016; 15: 235-246.

6. Lindquist LA, Go L, Fleisher J, et al. Relationship of health literacy to intentional and unintentional non-adherence of hospital discharge medications. J Gen Intern Med 2012; 27: 173-178.
7. Kutner M, Greenburg E, Jin Y, Paulsen C. The Health Literacy of America's Adults: Results from the 2003 National Assessment of Adult Literacy. NCES 2006-483. National Center for Education Statistics, 2006.

8. Cho YI, Lee S-YD, Arozullah AM, Crittenden KS. Effects of health literacy on health status and health service utilization amongst the elderly. Social Sci Med 2008; 66: 1809-1816.

9. Hugo M. Readability levels of emergency department's written discharge instructions, 1999.

10. Kleindl J. Reading ability of patients versus the readability of patient education material [dissertation]. North Dokota State University, North Dokota 2007.

11. McKenna K, Tooth L. Client education: a partnership approach for health practitioners: UNSW Press; 2006

12. Hoffmann T, Ladner Y. Assessing the suitability of written stroke materials: An evaluation of the interrater reliability of the suitability assessment of materials (SAM) checklist. Top Stroke Rehabil 2012; 19: 417-422.

13. Ahmadzadeh K, Khosravi A, arastoopoor S, Tahmasebi R. Assessing the Readability of Patient Education Materials about Diabetes Available in Shiraz Health Centers. Iran J Med Educ 2014; 14: 661-667.

14. Berk L. Development Through The Lifespan (4 ${ }^{\text {th }}$ int. ed). Pearson Education, USA 2006.

15. Doak CC, Doak LG, Root JH. Teaching patients with low literacy skills. Philadelphia 1985.

16. Rhee RL, Von Feldt JM, Schumacher HR, Merkel PA. Readability and suitability assessment of patient education materials in rheumatic diseases. Arthrit Care Res 2013; 65: 1702-1706.

17. Chedraui P, Blümel JE, Baron G, et al. Impaired quality of life among middle aged women: a multicentre Latin American study. Maturitas 2008; 61: 323-329.

18. Lu J, Liu J, Eden J. The experience of menopausal symptoms by Arabic women in Sydney. Climacteric 2007; 10: 72-79.

19. Peeyananjarassri K, Cheewadhanaraks S, Hubbard M, et al. Menopausa symptoms in a hospital-based sample of women in southern Thailand. Climacteric 2006; 9: 23-29.

20. Hassan Zadeh GR, Yekkezare S, Vaziri S, et al. Knowledge of women about menopause in Qazvin. J Qazvin Univ Med Sci 2003; 7: 21-25.

21. World Health Organization. Research on the menopause in the 1990s: report of a WHO scientific group. WHO, Geneva 1996.

22. SCo I. Iran Statistical Yearbook. Statistical Centre of Iran, Tehran 2015.

23. Krajewska-Ferishah K, Krajewska-Kulak E, Terlikowski S, Wiktor $\mathrm{H}$. Analysis of quality of life women in menopause period in Poland. Progress Health Sci 2011; 1: 52-59.

24. Froberg DG, Kane RL. Methodology for measuring health-state preferences - II: Scaling methods. J Clin Epidemiol 1989; 42: 459-471.

25. Michie S, Van Stralen MM, West R. The behaviour change wheel: a new method for characterising and designing behaviour change interventions. Implement Sci 2011; 6: 42.

26. Charbonneau DH. Health literacy and the readability of written information for hormone therapies. J Midwifery Womens Health 2013; 58 : 265-270.

27. Yoshany N, Bahri N, Morovati Sharif Abad MA, et al. Effects of Training the Menopausal Health on Knowledge and Performance of Husbands with Women during Transitional Period to Menopause. J Health 2018; 9: 27-35.

28. Bahri N, Yoshany N, Morowatisharifabad MA, et al. The effects of menopausal health training for spouses on women's quality of life during menopause transitional period. Menopause 2016; 23: 183-188.

29. Yoshany N, Morowatisharifabad MA, Mihanpour H, et al. The effect of husbands' education regarding menopausal health on marital satisfaction of their wives. J Menopausal Med 2017; 23: 15-24.

30. Lopez MH. Does Speaking a Second Language Affect Labor Market Outcomes? Evidence from National Adult Literacy Survey of 1992. School of Public Affairs, University of Maryland, Maryland 1999.

31. Gold J, Aitken C, Dixon H, et al. A randomised controlled trial using mobile advertising to promote safer sex and sun safety to young people. Health Educ Res 2011; 26: 782-794.

32. Doak C, Doak L, Root J. Suitability assessment of materials. SAM, Washington, DC 1994. 
33. Vallance JK, Taylor LM, Lavallee C. Suitability and readability assessment of educational print resources related to physical activity: Implications and recommendations for practice. Patient Educ Couns 2008; 72: $342-$ 349.

34. Weintraub D, Maliski SL, Fink A, et al. Suitability of prostate cancer education materials: applying a standardized assessment tool to currently available materials. Patient Educ Couns 2004; 55: 275-280.

35. Abdolmalaki B, Peyman N, Esmaeili H. Evaluation of health literacy rate of postmenopausal women covered by Mashhad health centers in 2018. J Health Lit 2018; 3: 182-190.

36. Buxton T. Effective ways to improve health education materials. J Health Educ 1999; 30: 47-61.

37. Sadeghi R, Mahmoodabad SSM, Fallahzadeh H, Rezaeian M, Bidaki R, Khanjani N. Readability and suitability assessment of adolescent education material in preventing hookah smoking. Int J High Risk Behav Addict 2019 (in press).

38. Walsh TM, Volsko TA. Readability assessment of internet-based consumer health information. Respir Care 2008; 53: 1310-1315.

39. Finnie RK, Felder TM, Linder SK, Mullen PD. Beyond reading level: a systematic review of the suitability of cancer education print and webbased materials. J Cancer Educ 2010; 25: 497-505.

40. Eames S, McKenna K, Worrall L, Read S. The suitability of written education materials for stroke survivors and their carers. Top Stroke Rehabil 2003; 10: 70-83. 